DFF 406-5-03

\title{
Fermion pairing in Bose-Fermi mixtures
}

\author{
F. Materd* \\ Dipartimento di Fisica, Università degli Studi di Firenze, \\ and Istituto Nazionale di Fisica Nucleare, Sezione di Firenze, \\ Via G. Sansone 1, I-50019, Sesto Fiorentino, (Firenze), Italy
}

\begin{abstract}
An effective interaction between fermions in a Bose-Fermi mixture is derived. It is induced by density fluctuations of the bosonic background. The contributions from states containing both one and two virtual phonons are taken into account self-consistently. The time dependence of the effective interaction has been removed by assuming that the velocity of the fermions at the Fermi surface is much larger than the sound velocity in the Bose gas. This assumption is more appropriate for the actual experimental situations than the usual approximation of neglecting retardation effects. The effective interaction turns out to be attractive and, as a consequence, can give rise to a superconducting phase in the Fermi component of the mixture. The fermions are considered in only one magnetic state, so that pairing can be effective only in odd- $l$ channels. It has been found that the onset of the superconducting phase can occur at temperatures $(>100 \mathrm{nK})$ of the same order of magnitude as the Fermi temperature $(\sim 300 \mathrm{nK})$, and the energy gap in the excitation spectrum is a small fraction $(\sim 1 \%)$ of the Fermi energy.
\end{abstract}

PACS numbers: 03.75.-b, 67.60.-g, 74.20.Fg

*Electronic address: matera@fi.infn.it 


\section{INTRODUCTION}

Effective fermion-fermion interactions induced by the exchange of virtual phonons in fermion-boson mixtures were already studied in the 1960s, in connection with dilute solutions of ${ }^{3} \mathrm{He}$ in superfluid ${ }^{4} \mathrm{He}$, see e.g. Ref. 1]. Recently, a renewed interest in this subject has arisen, following the actual availability of trapped atomic gases at a temperature sufficiently low to exhibit quantum properties. In particular, physical situations are reproduced, in which a highly condensed Bose gas is mixed with an almost degenerate Fermi gas and the interaction between these two gases can be studied at various values of temperature and density [2, 3, 4, 5]. On the theoretical side, particular attention has been devoted to the possibility of the onset of a supercoducting phase in the Fermi component of the mixture 6, 7, 8, 9]. For the fermion gases used in experiments the bare interatomic potential is repulsive. Then, the necessary attractive interaction between two fermions should be provided by the exchange of virtual phonons, through a mechanism similar to that responsible for the superconductivity phenomenon in metals. Investigations of the physical conditions for a a Cooper instability in the $s$-wave and $p$-wave channels have recently been performed [6, 7, 8, 9].

For a bare fermion-boson interaction with zero-range, the induced fermion-fermion interaction is essentially proportional to the correlator of density fluctuations of the bosonic background. In the references [1, 6, 7, 8, 9], the density fluctuations have been calculated taking into account only single- phonon intermediate states. In the present paper the contribution from two-phonon intermediate states is also included. This does not simply amount to an improvement of the approximation scheme, because two-phonon and onephonon states occur on the same basis in the evaluation of the effective fermion-fermion interaction, at lowest order in the fermion-boson coupling constant. Moreover, when the fractions of condensed and non-condensed components of the boson gas are comparable, the one-phonon and two-phonon contributions turn out to be of the same order of magnitude.

The induced interaction between fermions is given by the boson density-density correlator, evaluated for the time interval between two successive fermion-boson interactions. In order to simplify calculations, different approximations can be introduced to eliminate this time dependence. In Refs. [1, 6, 7, 8, 9] retardation effects have been neglected, always by setting the frequency to zero in the time-Fourier transform of the correlator. For a degenerate Fermi gas, this approximation can be justified if the Fermi velocity is much lower than the sound velocity in the Bose gas. In the present approach the opposite approximation is used: the correlator is taken at equal times, i.e. the induced interaction between two fermions is mediated by the static correlation function for boson density fluctuations. This approximation is more appropriate for the physical conditions obtained in the mixtures of trapped Bose-Fermi atoms produced in the laboratory [2, 3, 4, 5]. For these mixtures the density of the degenerate Fermi gas is a relevant fraction of the density of the Bose gas.

The aim of the present work is to assess the possible occurrence of a superconducting phase in the Fermi component of an ultracold mixture of Bose-Fermi gases, for values of density and temperature obtained in actual experiments, and to give an estimate of the gap in the spectrum of excitation energies. Although all the experiments have been performed with harmonic-oscillator traps, a spatially homogeneous system is considered. In this case, calculations are considerably simplified and compact analytical expressions can be derived.

At the densities considered in the experiments, for the two species of gases we can assume that the interatomic interactions have a short range and mainly occur in the $s$-wave channel. 
Moreover, according to the experimental situations, both the Fermi and Bose atoms are considered in only one internal state. In this case, the Pauli principle allows us to neglect the bare interaction between fermions, at least in a mean-field treatment. In fact, in the zerorange limit for the interaction, the contributions from the direct ( Hartree ) and exchange ( Fock ) terms of the mean-field cancel each-other exactly, while short range correlations should not play an important role for a sufficiently dilute Fermi gas, i.e. for $a_{F F}^{3} n_{F}<<1$ where $a_{F F}$ is the scattering length and $n_{F}$ is the fermion density. Concerning the Cooper condensation, it can occur only for odd values of the orbital angular momentum for a spinaligned couple of fermions, so that there is no contribution from a $s$-wave interaction.

Experiments have been performed with gases, which show repulsive boson-boson interactions [3, 5], this ensures the stability of the mixture towards separation of the two species of gases allowing us to study mixtures with a sufficiently high number of atoms. Moreover, the interaction between a fermion and a boson in the mixture ${ }^{40} \mathrm{~K}-{ }^{87} \mathrm{Rb}$ of the Florence experiment [5] is strongly attractive, thus the induced fermion-fermion interaction can be very effective to bind fermions in correlated pairs. The calculations are performed on a general ground, however, when more phenomenological aspects are concerned, they mostly refer to the physical environment of Ref. [5].

\section{FORMALISM}

In this section the effective interaction between two fermions, mediated by density fluctuations of the Bose gas, and the equation for the energy gap in the excitation spectrum of the Fermi gas are derived. The calculations are performed by means of functional methods thoroughly, since both the properties of the Bose condensate and the induced fermion-fermion interaction can be directly obtained in the same scheme ( for a introduction to functional methods see e.g. Ref. [10, 11] ).

\section{A. Effective interaction}

The starting point is the functional-integral expression for the grand-canonical partition function of the Bose-Fermi mixture, that reads

$$
Z=\int \mathcal{D}\left(\phi, \phi^{*}, \psi, \psi^{*}\right) \exp \left[-\left(S_{B}\left(\phi, \phi^{*}\right)+S_{F}\left(\psi, \psi^{*}\right)+S_{I}\left(\phi, \phi^{*}, \psi, \psi^{*}\right)\right)\right] .
$$

The integration is performed over the complex field $\phi(\mathbf{x}, \tau)$ (bosons ) and the Grassman field $\psi(\mathbf{x}, \tau)$ (fermions ), which are respectively periodic and anti-periodic in the imaginary-time interval $(0, \beta=1 / T)$ ( units such that $\hbar=c=k_{B}=1$ are used ). Since we deal with spinpolarized particles, $\phi(\mathbf{x}, \tau)$ and $\psi(\mathbf{x}, \tau)$ are one-component fields. The action functional has been written as the sum of three terms: the action for the boson field

$$
S_{B}\left(\phi, \phi^{*}\right)=\int_{0}^{\beta} d \tau \int d \mathbf{x}\left[\phi^{*}(\mathbf{x}, \tau)\left(\frac{\partial}{\partial \tau}-\frac{\nabla^{2}}{2 m_{B}}-\mu_{B}\right) \phi(\mathbf{x}, \tau)+\frac{1}{2} \gamma|\phi(\mathbf{x}, \tau)|^{4}\right],
$$

the action for the fermion field

$$
S_{F}\left(\psi, \psi^{*}\right)=\int_{0}^{\beta} d \tau \int d \mathbf{x}\left[\psi^{*}(\mathbf{x}, \tau)\left(\frac{\partial}{\partial \tau}-\frac{\nabla^{2}}{2 m_{F}}-\mu_{F}\right) \psi(\mathbf{x}, \tau)\right]
$$


and the interaction term containing both fields

$$
S_{I}\left(\phi, \phi^{*}, \psi, \psi^{*}\right)=\lambda \int_{0}^{\beta} d \tau \int d \mathbf{x}\left[\psi^{*}(\mathbf{x}, \tau) \psi(\mathbf{x}, \tau) \phi^{*}(\mathbf{x}, \tau) \phi(\mathbf{x}, \tau)\right] .
$$

Here $\mu_{B}$ and $\mu_{F}$ denote the boson and fermion chemical potentials respectively. In writing the action functional, the usual zero-range approximation for the interactions between atoms has been adopted [6, 7, 8, 9]. The corresponding coupling constants are defined as follows: $\gamma=4 \pi a_{B B} / m_{B}$ and $\lambda=2 \pi a_{B F} / m_{R}$, where $a_{B B}$ and $a_{B F}$ are the boson-boson and fermionboson scattering lengths respectively, and $m_{R}=m_{B} m_{F} /\left(m_{B}+m_{F}\right)$ is the reduced mass for a boson of mass $m_{B}$ and a fermion of mass $m_{F}$. Finally, according to the previous remarks, in Eq. (3) the fermion-fermion interaction has been omitted.

The Bose gas is treated within the Gaussian approximation, i.e. all terms of order higher than second in the fluctuations of the boson field about the uniform and constant condensate amplitude are neglected. This corresponds to the Bogoliubov approximation for a simple Bose gas. For a weakly interacting gas, $n_{B} a_{B B}^{3}<<1$ ( $n_{B}$ is the boson density ) and at low temperatures, this approximation is well justified [12]. The first step consists in evaluating the condensate amplitude about which the perturbative expansion can be performed. The standard methods employed for a simple Bose gas [10, 11] are extended to a fermion-boson mixture. First the action functional is integrated over the fermion fields, then, a uniform and constant boson field $\phi(\mathbf{x}, \tau)=\varphi$, which makes the action stationary, is searched for. The integration over the fermion fields can be performed explicitely, since these fields occur with a Gaussian form in the functional integral. This gives the two following contributions to the action functional

$$
-\operatorname{Tr} \ln G_{0}^{-1}\left(\mathbf{x}-\mathbf{x}^{\prime}, \tau-\tau^{\prime}\right)-\operatorname{Tr} \ln \left(\mathbf{1}+\lambda G_{0}^{-1}\left(\mathbf{x}-\mathbf{x}^{\prime}, \tau-\tau^{\prime}\right) \phi(\mathbf{x}, \tau) \phi^{*}(\mathbf{x}, \tau)\right),
$$

where the trace operation implies integration over the continuous variables $\mathbf{x}, \tau$. Here $G_{0}^{-1}\left(\mathbf{x}-\mathbf{x}^{\prime}, \tau-\tau^{\prime}\right)$ is the inverse of the Green function for free fermions. The first variation of the action functional takes the contribution

$$
-\lambda \phi(\mathbf{x}, \tau) G\left(\mathbf{x}, \mathbf{x}, \tau, \tau+0^{+}\right)=\lambda \phi(\mathbf{x}, \tau) n_{F}
$$

from the second term of Eq. (15). The quantity $G\left(\mathbf{x}, \mathbf{x}^{\prime}, \tau, \tau^{\prime}\right)$ is the Green function for fermions interacting with the scalar field $\phi(\mathbf{x}, \tau)$. Adding the contributions coming from the boson term of the action, for the density of the zero-momentum Bose condensate, $n_{B}^{0}=\varphi^{2}$, the following equation is obtained

$$
n_{B}^{0}=\frac{\mu_{B}-\lambda n_{F}}{\gamma} .
$$

This equation has been derived assuming, without loss of generality, that the quantity $\varphi$ is real. We can see that the effect of the interaction with the Fermi gas simply amounts to replacing the chemical potential $\mu_{B}$ with the effective value $\mu_{B}-\lambda n_{F}$.

The next step is the introduction of the change of variables $\phi^{\prime}=\phi-\varphi$ in the terms $S_{B}\left(\phi, \phi^{*}\right)$ and $S_{I}\left(\phi, \phi^{*}, \psi, \psi^{*}\right)$ of the action functional. The term with $\varphi^{2}$ represents the interaction of the fermions with the condensate mean-field, $\lambda n_{B}^{0}$ and can be added to the chemical potential $\mu_{F}$ in $S_{F}\left(\psi, \psi^{*}\right)$. The terms containing products of more than two amplitudes $\phi^{\prime}$, are neglected according to the Bogoliubov approximation. Thus, the action for 
the fluctuations of the boson field is given by

$$
\begin{aligned}
S^{(2)}\left(\phi^{\prime}, \phi^{\prime *}, \psi, \psi^{*}\right)= & \frac{1}{2} \int_{0}^{\beta} d \tau d \tau^{\prime} \int d \mathbf{x} d \mathbf{x}^{\prime} \hat{\phi}^{\prime \dagger}(\mathbf{x}, \tau) \widehat{D}^{-1}\left(\mathbf{x}, \mathbf{x}^{\prime}, \tau, \tau^{\prime}\right) \hat{\phi}^{\prime}\left(\mathbf{x}^{\prime}, \tau^{\prime}\right) \\
& +\frac{1}{2} \int_{0}^{\beta} d \tau \int d \mathbf{x}\left(\hat{\phi}^{\prime \dagger}(\mathbf{x}, \tau) \hat{\Gamma}(\mathbf{x}, \tau)+\text { c.c. }\right),
\end{aligned}
$$

where the vector field

$$
\hat{\phi}^{\prime}(\mathbf{x}, \tau)=\left(\begin{array}{c}
\phi^{\prime}(\mathbf{x}, \tau) \\
\phi^{\prime *}(\mathbf{x}, \tau)
\end{array}\right)
$$

the source term

$$
\hat{\Gamma}(\mathbf{x}, \tau)=\lambda \varphi\left(\begin{array}{c}
\psi^{*}(\mathbf{x}, \tau) \psi(\mathbf{x}, \tau) \\
\psi^{*}(\mathbf{x}, \tau) \psi(\mathbf{x}, \tau)
\end{array}\right)
$$

and the inverse of the Green function for the fluctuations $\phi^{\prime}$

$$
\widehat{D}^{-1}\left(\mathbf{x}, \mathbf{x}^{\prime}, \tau, \tau^{\prime}\right)=\widehat{D}_{0}^{-1}\left(\mathbf{x}, \mathbf{x}^{\prime}, \tau, \tau^{\prime}\right)+\widehat{V}\left(\mathbf{x}, \mathbf{x}^{\prime}, \tau, \tau^{\prime}\right)
$$

are introduced with the inverse of the Bogoliubov propagator

$$
\widehat{D}_{0}^{-1}\left(\mathbf{x}, \mathbf{x}^{\prime}, \tau, \tau^{\prime}\right)=\left(\begin{array}{cc}
\frac{\partial}{\partial \tau}-\frac{\nabla^{2}}{2 m_{B}}+\gamma n_{B}^{0} & \gamma n_{B}^{0} \\
\gamma n_{B}^{0} & -\frac{\partial}{\partial \tau}-\frac{\nabla^{2}}{2 m_{B}}+\gamma n_{B}^{0}
\end{array}\right) \delta\left(\tau-\tau^{\prime}\right) \delta\left(\mathbf{x}-\mathbf{x}^{\prime}\right)
$$

and the coupling matrix

$$
\widehat{V}\left(\mathbf{x}, \mathbf{x}^{\prime}, \tau, \tau^{\prime}\right)=\lambda\left(\begin{array}{cc}
\psi^{*}(\mathbf{x}, \tau) \psi(\mathbf{x}, \tau) & 0 \\
0 & \psi^{*}(\mathbf{x}, \tau) \psi(\mathbf{x}, \tau)
\end{array}\right) \delta\left(\tau-\tau^{\prime}\right) \delta\left(\mathbf{x}-\mathbf{x}^{\prime}\right) .
$$

In order to obtain an effective interaction for the fermions, the integration over the fluctuations $\phi^{\prime}$ and $\phi^{*}$ should be performed. The result is an effective action containing only the fermionic fields $\psi$ and $\psi^{*}$

$$
\begin{aligned}
S_{e f f}\left(\psi, \psi^{*}\right)= & \int_{0}^{\beta} d \tau \int d \mathbf{x}\left[\psi^{*}(\mathbf{x}, \tau)\left(\frac{\partial}{\partial \tau}-\frac{\nabla^{2}}{2 m_{F}}-\left(\mu_{F}-\lambda n_{B}^{0}\right)\right) \psi(\mathbf{x}, \tau)\right] \\
& +\frac{1}{2} \ln \left(\operatorname{det} \widehat{D}_{0}^{-1}\right)+\frac{1}{2} \ln \left[\operatorname{det}\left(\mathbf{1}+\widehat{D_{0}} \widehat{V}\right)\right] \\
& -\frac{1}{2} \int_{0}^{\beta} d \tau d \tau^{\prime} \int d \mathbf{x} d \mathbf{x}^{\prime} \hat{\Gamma}^{\dagger}(\mathbf{x}, \tau) \widehat{D}\left(\mathbf{x}, \mathbf{x}^{\prime}, \tau, \tau^{\prime}\right) \hat{\Gamma}\left(\mathbf{x}^{\prime}, \tau^{\prime}\right) .
\end{aligned}
$$

The term $\ln \left(\operatorname{det} \widehat{D}_{0}^{-1}\right)$ together with the action evaluated at the stationary point $\phi^{\prime}=0$, give the grand potential in the Bogoliubov approximation for a Bose gas with the effective chemical potential $\mu_{B}-\lambda n_{F}$.

The last two terms on right hand side of Eq. (8) represent an effective interaction among fermions. When expanded in powers of the fermion fields, both terms give rise to $n$-body forces. Only terms containing up to four fields are taken into account, so both states with more than two virtual phonons in the boson-density fluctuations and $n$-body forces among fermions, with $n$ greater than two, are neglected. We expect this to be a reasonable approximation for sufficiently dilute gases. According to this approximation, the boson 
Green function is replaced with the Bogoliubov propagator, $\widehat{D}=\widehat{D}_{0}$ in the source term, and terms up to second order are retained in the expansion

$$
\frac{1}{2} \operatorname{Tr} \ln \left(\mathbf{1}+\widehat{D}_{0} \widehat{V}\right)=-\frac{1}{2} \sum_{n} \frac{(-1)^{n}}{n} \operatorname{Tr}\left(\widehat{D}_{0} \widehat{V}\right)^{n}
$$

The space-Fourier transform of the contribution from the source term of Eq. (8) to the effective interaction between fermions is given by

$$
V_{1}\left(q, \tau_{1}-\tau_{2}\right)=-\lambda^{2} n_{B}^{0}\left[D_{0}^{(11)}\left(q, \tau_{1}-\tau_{2}\right)+D_{0}^{(11)}\left(q, \tau_{2}-\tau_{1}\right)+2 D_{0}^{(12)}\left(q, \tau_{1}-\tau_{2}\right)\right],
$$

where $D_{0}^{(i j)}$ are the elements of the $2 \times 2$ matrix $\widehat{D}_{0}$; for their explicit expressions see, e.g., Ref. [12] ( the definition of $\widehat{D}_{0}$ used here differs from that of Ref. [12] by an overall minus sign ). Concerning the contributions from the expansion of Eq. (9), the term with $n=1$ represents the mean field acting on the fermions due to the noncondensate fraction of the Bose gas. This term can be added to the chemical potential of the fermions, giving together with the analogous term of Eq. (8) the effective chemical potential $\mu_{F}^{*}=\mu_{F}-\lambda n_{B}$ for the fermions. The space-Fourier transform of the contribution from the term with $n=2$ reads

$$
\begin{aligned}
V_{2}\left(q, \tau_{1}-\tau_{2}\right)=-\lambda^{2} \int \frac{d \mathbf{k}}{(2 \pi)^{3}} \quad & {\left[D_{0}^{(11)}\left(k, \tau_{1}-\tau_{2}\right) D_{0}^{(11)}\left(\mathbf{k}-\mathbf{q}, \tau_{2}-\tau_{1}\right)\right.} \\
& \left.+D_{0}^{(12)}\left(k, \tau_{1}-\tau_{2}\right) D_{0}^{(12)}\left(\mathbf{k}-\mathbf{q}, \tau_{2}-\tau_{1}\right)\right]
\end{aligned}
$$

This term is the contribution to the effective interaction from states containing two virtual phonons.

A rough estimate of the terms of Eq. (9) corresponding to the exchange of $n>2$ virtual phonons between fermions can be done by means of the following considerations. The expansion of Eq. (9) contains correlations of the boson density fluctuations of higher and higher order when $n$ increases. According to the basic assumption made in this paper, the correlations will be evaluated at equal times. In this case, we should expect that the contribution of a term of the series with respect to that of the previous term, contains an extra power of $\lambda n_{B}^{\prime}$. Here, $n_{B}^{\prime}$ is the density of the noncondensate bosons. In addition, when $n$ increases by a unit, the effective action of Eq. (8) contains an additional integration on the time, which gives a multiplicative factor of the order of magnitude as $\beta$. Thus, we can interpret the right hand side of Eq. (9) as a perturbative expansion in powers of the adimensional parameter $\lambda n_{B}^{\prime} / T$. Similar considerations can be done for the terms neglected in the perturbative expansion of the last term of Eq. (9). At the densities and temperatures considered in this paper the parameter $\left|\lambda n_{B}^{\prime} / T\right|$ takes a value of $\sim 0.15$. Since the order parameter of the superconducting phase, in the approach of this paper turns out to be not critically dependent on the fermion-fermion interaction ( see Eq. (23) of Sec. III), corrections to the effective fermion-fermion interaction coming from terms of order higher than second will be neglected in order to simplify calculations.

\section{B. Pairing field}

The effective interaction obtained involves fermions at two different times. The approximations which can be introduced in order to get rid of the retardation effects and simplify 
calculations, will be discussed later. Now, the equations for the effective field representing the pairing of two fermions, are derived.

With the approximations discussed above, the partition function of Eq. (1I) assumes the following factorized form

$$
Z=\exp \left(-\beta \Omega_{B}\right) \int \mathcal{D}\left(\psi, \psi^{*}\right) \exp \left[-S_{e f f}\left(\psi, \psi^{*}\right)\right]
$$

where $\Omega_{B}$ is the grand potential of the Bose gas, while the fermion effective action of Eq. (8) becomes

$$
\begin{aligned}
S_{e f f}\left(\psi, \psi^{*}\right)= & \int_{0}^{\beta} d \tau \int d \mathbf{k}\left[\psi^{*}(\mathbf{k}, \tau)\left(\frac{\partial}{\partial \tau}+\frac{k^{2}}{2 m_{F}}-\mu_{F}^{*}\right) \psi(\mathbf{k}, \tau)\right] \\
& +\frac{1}{2(2 \pi)^{3}} \int_{0}^{\beta} d \tau_{1} d \tau_{2} \int \prod_{i} d \mathbf{k}_{i} \delta\left(\mathbf{k}_{1}+\mathbf{k}_{2}-\mathbf{k}_{3}-\mathbf{k}_{4}\right) \\
& \times\left[V_{e f f}\left(\mathbf{k}_{3}-\mathbf{k}_{1}, \tau_{1}-\tau_{2}\right) \psi^{*}\left(\mathbf{k}_{1}, \tau_{1}\right) \psi^{*}\left(\mathbf{k}_{2}, \tau_{2}\right) \psi\left(\mathbf{k}_{4}, \tau_{2}\right) \psi\left(\mathbf{k}_{3}, \tau_{1}\right)\right],
\end{aligned}
$$

with $V_{\text {eff }}\left(\mathbf{k}_{3}-\mathbf{k}_{1}, \tau_{1}-\tau_{2}\right)=V_{1}\left(\left|\mathbf{k}_{3}-\mathbf{k}_{1}\right|, \tau_{1}-\tau_{2}\right)+V_{2}\left(\left|\mathbf{k}_{3}-\mathbf{k}_{1}\right|, \tau_{1}-\tau_{2}\right)$.

The pairing field is identified with the composite field which makes the effective action $S_{\text {eff }}\left(\psi, \psi^{*}\right)$ stationary. This can be determined by means of a Stratonovich-Hubbard transformation [13, 14] introducing auxiliary fields for the couples $\psi \psi$ and $\psi^{*} \psi^{*}$, and by equating to zero the first variation of the resulting effective action with respect to the auxiliary fields. After the transformation, the exponential in the integral of Eq. (12) is replaced by

$$
\begin{aligned}
\exp \left[-S_{e f f}\left(\psi, \psi^{*}\right)\right]= & \exp \left[-\int_{0}^{\beta} d \tau \int d \mathbf{k} \psi^{*}(\mathbf{k}, \tau)\left(\frac{\partial}{\partial \tau}+\frac{k^{2}}{2 m_{F}}-\mu_{F}^{*}\right) \psi(\mathbf{k}, \tau)\right] \\
& \times \int \mathcal{D}\left(\Delta^{*}, \Delta\right) \exp \left[\int_{0}^{\beta} \prod_{i} d \tau_{i} \int \prod_{i} d \mathbf{k}_{i}\right. \\
& \frac{1}{2}\left[\Delta^{*}\left(\mathbf{k}_{1}, \mathbf{k}_{2}, \tau_{1}, \tau_{2}\right) V_{e f f}^{-1}\left(\mathbf{k}_{1}, \mathbf{k}_{2}, \tau_{1}, \tau_{2} ; \mathbf{k}_{3}, \mathbf{k}_{4}, \tau_{3}, \tau_{4}\right) \Delta\left(\mathbf{k}_{3}, \mathbf{k}_{4}, \tau_{3}, \tau_{4}\right)\right] \\
& +\frac{1}{2} \int d \mathbf{k}_{1} d \mathbf{k}_{2} \int d \tau_{1} d \tau_{2}\left[\Delta^{*}\left(\mathbf{k}_{1}, \mathbf{k}_{2}, \tau_{1}, \tau_{2}\right) \psi\left(\mathbf{k}_{1}, \tau_{1}\right) \psi\left(\mathbf{k}_{2}, \tau_{2}\right)\right. \\
& \left.\left.+\psi^{*}\left(\mathbf{k}_{1}, \tau_{1}\right) \psi^{*}\left(\mathbf{k}_{2}, \tau_{2}\right) \Delta\left(\mathbf{k}_{1}, \mathbf{k}_{2}, \tau_{1}, \tau_{2}\right)\right]\right]
\end{aligned}
$$

where $\Delta$ and $\Delta^{*}$ are bosonic auxiliary fields and the matrix

$$
V_{e f f}^{-1}\left(\mathbf{k}_{1}, \mathbf{k}_{2}, \tau_{1}, \tau_{2} ; \mathbf{k}_{3}, \mathbf{k}_{4}, \tau_{3}, \tau_{4}\right)
$$

is the inverse of the effective interaction

$$
\begin{gathered}
<\mathbf{k}_{1} \mathbf{k}_{2}\left|V_{e f f}\left(\tau_{1}, \tau_{2} ; \tau_{3}, \tau_{4}\right)\right| \mathbf{k}_{3} \mathbf{k}_{4}>= \\
\frac{1}{(2 \pi)^{3}} V_{e f f}\left(\mathbf{k}_{3}-\mathbf{k}_{1}, \tau_{1}-\tau_{2}\right) \delta\left(\tau_{3}-\tau_{1}\right) \delta\left(\tau_{4}-\tau_{2}\right) \delta\left(\mathbf{k}_{1}+\mathbf{k}_{2}-\mathbf{k}_{3}-\mathbf{k}_{4}\right) .
\end{gathered}
$$

In the functionals containing the coupling between fermions and auxiliary fields, only the parts of $\Delta$ and $\Delta^{*}$ which are antisymmetric under the permutation $1 \leftrightarrow 2$ survive. This reflects the prescription of the Pauli principle for fermions in the same spin state. 
The integration over the fermionic fields can be formally performed, giving the following action for the auxiliary fields

$$
\begin{aligned}
S\left(\Delta, \Delta^{*}\right)=-\int_{0}^{\beta} \prod_{i} d \tau_{i} \int \prod_{i} d \mathbf{k}_{i} \frac{1}{2}[\quad & \Delta^{*}\left(\mathbf{k}_{1}, \mathbf{k}_{2}, \tau_{1}, \tau_{2}\right) V_{e f f}^{-1}\left(\mathbf{k}_{1}, \mathbf{k}_{2}, \tau_{1}, \tau_{2} ; \mathbf{k}_{3}, \mathbf{k}_{4}, \tau_{3}, \tau_{4}\right) \\
& \left.\times \Delta\left(\mathbf{k}_{3}, \mathbf{k}_{4}, \tau_{3}, \tau_{4}\right)\right]-\frac{1}{2} \operatorname{Tr} \ln \left(\mathbf{1}-\widehat{\mathcal{G}_{0}} \widehat{\Delta}\right)
\end{aligned}
$$

where $\widehat{\mathcal{G}}_{0}$ is a $2 \times 2$ diagonal matrix, whose elements are the propagator for independent fermions with the effective chemical potential $\mu_{F}^{*}$, and the matrix $\widehat{\Delta}$ is given by

$$
\widehat{\Delta}\left(\mathbf{k}_{1}, \mathbf{k}_{2}, \tau_{1}, \tau_{2}\right)=\left(\begin{array}{cc}
0 & \Delta\left(\mathbf{k}_{1}, \mathbf{k}_{2}, \tau_{1}, \tau_{2}\right) \\
\Delta^{*}\left(\mathbf{k}_{1}, \mathbf{k}_{2}, \tau_{1}, \tau_{2}\right) & 0
\end{array}\right) .
$$

The equation for the pairing field can be obtained by equating to zero the functional derivative of $S\left(\Delta, \Delta^{*}\right)$ with respect to either $\Delta^{*}$ or $\Delta$, equivalently. It reads

$$
\begin{aligned}
\Delta\left(\mathbf{k}, \mathbf{k}^{\prime}, \tau-\tau^{\prime}\right)=\frac{1}{(2 \pi)^{3}} \int d \mathbf{k}_{1} d \mathbf{k}_{2} \quad & V_{e f f}\left(\mathbf{k}_{1}-\mathbf{k}, \tau-\tau^{\prime}\right) \delta\left(\mathbf{k}+\mathbf{k}^{\prime}-\mathbf{k}_{1}-\mathbf{k}_{2}\right) \\
& \times \mathcal{G}^{(12)}\left(\mathbf{k}_{2}, \mathbf{k}_{1}, \tau-\tau^{\prime}\right),
\end{aligned}
$$

where $\mathcal{G}^{(12)}\left(\mathbf{k}_{2}, \mathbf{k}_{1}, \tau-\tau^{\prime}\right)$ is the anomalous propagator for fermions interacting with the pairing field $\widehat{\Delta}$ ( see, e.g., Ref. [12] ). Because of time-translation invariance both the pairing field and the fermion propagator depend only on the difference of times.

It is convenient to introduce the center of mass momentum $\mathbf{P}=\mathbf{k}+\mathbf{k}^{\prime}=\mathbf{k}_{1}+\mathbf{k}_{2}$, thus, for a given value of $\mathbf{P}$, an equation containing only one integration variable, $\mathbf{k}$, can be obtained. The solutions of Eq. (16) with $\mathbf{P} \neq 0$, correspond to a breaking of the space-translational symmetry, i.e. the LOFF phase [15]. Here, only solutions with vanishing center of mass momentum are considered. In this case, the pairing field and the anomalous propagator depend on only one momentum, $\mathbf{k}=-\mathbf{k}^{\prime}$ and $\mathbf{k}_{1}=-\mathbf{k}_{2}$, respectively. The propagator $\mathcal{G}^{(12)}\left(\mathbf{k}_{1}, \tau-\tau^{\prime}\right)$ is given by the Fourier series

$$
\mathcal{G}^{(12)}\left(\mathbf{k}_{1}, \tau-\tau^{\prime}\right)=-\frac{1}{\beta} \sum_{n} \frac{e^{-i \omega_{n}\left(\tau-\tau^{\prime}\right)} \Delta\left(\mathbf{k}_{1}, \omega_{n}\right)}{\omega_{n}^{2}+\xi_{k_{1}}^{2}+\Delta\left(\mathbf{k}_{1}, \omega_{n}\right) \Delta^{*}\left(\mathbf{k}_{1}, \omega_{n}\right)},
$$

where $\omega_{n}$ are the Matsubara frequencies for fermions and $\xi_{k_{1}}^{2}=\left(k_{1}^{2} / 2 m_{F}-\mu_{F}^{*}\right)^{2}$.

Now, two different paths of approximation which allow us to neglect the time (or frequency ) dependence of the pairing field, are discussed. Generally, for evaluating the time behavior of a propagator we should make an analytical continuation to real times $(-i \tau \rightarrow t)$ or, equivalently to real frequencies $\left(i \omega_{n} \rightarrow \omega\right)$. However, for the two cases that will be envisaged, we can directly refer to the equations just derived. Both approximations are based on the comparison of the characteristic times of propagation for fermions and for bosons. For a quasi degenerate Fermi gas only the particles with momenta lying about the Fermi surface partecipate in the pairing process. Then, for a given momentum $q$, the fermion time-scale can be estimated as $t_{F}=1 / v_{F} q$. While for bosons, the quantity $t_{B}=1 / c_{s} q$, where $c_{s}$ is the sound velocity in the Bose gas, can be assumed to give an estimate of the characteristic time. The comparison between the two time-scales reduces to compare the velocities $v_{F}$ and $c_{s}$. The case, when $c_{s}>>v_{F}$, is examined first. This is the assumption 
usually made when studying boson-induced interactions between fermions for Bose-Fermi mixtures. For $c_{s}>>v_{F}$ retardation effects on the induced interaction can be neglected, i.e. only the $\omega=0$ component of the time-Fourier transform of the boson propagators in Eqs. (10) and (11) is retained. Also, the fermion propagator $\mathcal{G}^{(12)}\left(\mathbf{k}, \tau-\tau^{\prime}\right)$ can be evaluated at equal times $\left(\tau=\tau^{\prime}\right)$. The pairing field in turn becomes an instantaneous function of the time difference, $\Delta\left(\mathbf{k}, \tau-\tau^{\prime}\right)=\Delta(\mathbf{k}) \delta\left(\tau-\tau^{\prime}\right)$. The equation obeyed by the pairing field is the usual gap equation of the BCS theory. The second approximation is the opposite of that just discussed: the Fermi velocity can be assumed to be much larger than the sound velocity. Then, the propagator $\mathcal{G}^{(12)}\left(\mathbf{k}, \tau-\tau^{\prime}\right)$ is a fast changing function of time with respect to the boson propagators, so that in Eq. (16) the static limit of the effective interaction can be taken. In this case, the boson-induced interaction is the space-Fourier transform ( times $-\lambda^{2}$ ) of the static correlation function for density fluctuations. In the following calculations, this second approximation will be adopted, since it is closer to the physical situations realized in experiments. Thus, in the frequency representation, the equation (16) for the pairing field with $\mathbf{P}=0$, is given by the following expression

$$
\Delta\left(\mathbf{k}, \omega_{n}\right)=-\int \frac{d \mathbf{k}_{1}}{(2 \pi)^{3}} \frac{V_{e f f}\left(\mathbf{k}-\mathbf{k}_{1}, \tau=\tau^{\prime}\right) \Delta\left(\mathbf{k}_{1}, \omega_{n}\right)}{\omega_{n}^{2}+\xi_{k_{1}}^{2}+\left|\Delta\left(\mathbf{k}_{1}, \omega_{n}\right)\right|^{2}} .
$$

When evaluating the energy gap in the spectrum of elementary excitations, an analytical continuation of this equation to real frequencies should be made, in order to determine the real poles of the fermion Green function. However, for assessing the occurrence of a superconducting phase and for evaluating the order of magnitude of the energy gap, it is sufficient to take into account only the $\omega=0$ component of the pairing field, $\Delta(\mathbf{k})$. This quantity satisfies the equation

$$
\Delta(\mathbf{k})=-\int \frac{d \mathbf{k}_{1}}{(2 \pi)^{3}} \frac{V_{e f f}\left(\mathbf{k}-\mathbf{k}_{1}\right) \Delta\left(\mathbf{k}_{1}\right)}{\xi_{k_{1}}^{2}+\left|\Delta\left(\mathbf{k}_{1}\right)\right|^{2}}
$$

Here $V_{\text {eff }}\left(\mathbf{k}-\mathbf{k}_{1}\right)$ denotes the effective interaction at equal times, its explicit expression, with $q=\left|\mathbf{k}-\mathbf{k}_{1}\right|$, is

$$
\begin{aligned}
V_{e f f}(q)= & -\lambda^{2} n_{B}^{0}\left[1+2 n_{B}^{\prime}(q)-\frac{\gamma n_{B}^{0}}{E_{q}}[1+2 \tilde{n}(q)]\right]-\lambda^{2} \int \frac{d \mathbf{k}}{(2 \pi)^{3}}\left[n_{B}^{\prime}(k)\left[1+n_{B}^{\prime}(|\mathbf{k}-\mathbf{q}|)\right]+\right. \\
& \left.+\frac{\left(\gamma n_{B}^{0}\right)^{2}}{4 E_{k} E_{|\mathbf{k}-\mathbf{q}|}}[1+2 \tilde{n}(k)][1+2 \tilde{n}(|\mathbf{k}-\mathbf{q}|)]\right]
\end{aligned}
$$

where $E_{k}$ are the excitation energies of the Bose gas calculated within the Bogoliubov approximation, $E_{k}=\sqrt{\left(k^{2} /\left(2 m_{B}\right)\right)^{2}+2 \gamma n_{B}^{0} k^{2} /\left(2 m_{B}\right)}, n_{B}^{\prime}(k)$ the occupation numbers of noncondensate bosons, and

$$
\tilde{n}(k)=\frac{1}{e^{\beta E_{k}}-1} .
$$

The first term on right hand side of Eq. (20) is the contribution from states containing one virtual phonon, and it comes from the correlations of the products of the condendate and noncondensate amplitudes taken at two different space points. The second term is the contribution from states containing two virtual phonons, and it is related to the noncondensate-density correlations.

A few remarks should be made. First, according to the Pauli principle, the pairing field should be an odd function of $\mathbf{k}$ in order to change sign under the permutation of the two 
correlated fermions. This excludes contributions to the pairing field from interactions acting only in the $s$-wave channel. So that, as previously remarked, the bare fermion-fermion interaction can be completely neglected in the present scheme. In addition, the function $V_{\text {eff }}(q)$ has a simple pole, when $q \rightarrow 0$. However, this is a fictitious pole, since, if the the integrals in Eq. (20) are replaced by a sum over the momenta, the boson states with vanishing momentum should be excluded. Actually, this restriction may be omitted, because it affects the integrand of Eq. (19) only at a single point and the integration gives a finite result. Finally, it is worth noting that $V_{e f f}(q)$ does not represent a usual two-body potential, since an integration over the time is absent.

In order to solve Eq. (19) it is convenient to expand $\Delta(\mathbf{k})$ in spherical harmonics

$$
\Delta(\mathbf{k})=\sum_{l, m} \sqrt{\frac{4 \pi}{2 l+1}} \Delta_{l, m}(k) Y_{l}^{m}\left(\Omega_{k}\right),
$$

with only odd values of $l$ contributing. To simplify calculations, an angle-average approximation for $\left|\Delta\left(\mathbf{k}_{1}\right)\right|^{2}$ can be made

$$
\left|\Delta\left(\mathbf{k}_{1}\right)\right|^{2} \rightarrow \bar{\Delta}^{2}\left(k_{1}\right)=\sum_{l, m} \frac{1}{2 l+1}\left|\Delta_{l, m}\left(k_{1}\right)\right|^{2} .
$$

With this approximation, the different $m$-components turn out to be uncoupled and all equal; in addition, they can be assumed to be real and positive, without loss of generality. The quantity $\bar{\Delta}^{2}(k)$ represents the averaged energy gap in the quasiparticle spectrum of the superconducting phase.

The equations for the partial waves of the pairing field,

$$
\Delta_{l}(k)=-\int \frac{d k_{1}}{4 \pi^{2}} k_{1}^{2} \frac{V_{l}\left(k, k_{1}\right) \Delta_{l}\left(k_{1}\right)}{\xi_{k_{1}}^{2}+\bar{\Delta}^{2}\left(k_{1}\right)},
$$

can still be coupled via the term $\bar{\Delta}^{2}\left(k_{1}\right)$. Here, the components $V_{l}\left(k, k_{1}\right)$ are defined as

$$
V_{l}\left(k, k_{1}\right)=\int d x V_{e f f}\left(k^{2}+k_{1}^{2}-2 k k_{1} x\right) P_{l}(x) .
$$

They are bounded quantities for $k=k_{1}$, in spite of the occurence of a pole when the argument of $V_{\text {eff }}(q)$ vanishes.

\section{RESULTS}

The temperature, density and relative concentrations of the gas mixture are assumed such that a relevant fraction of the Bose gas is in the condensate phase and the Fermi gas is still quite degenerate. To be more specific, we refer to the experimental environment described in Ref. [5], where a mixture of ${ }^{87} \mathrm{Rb}$ (bosons) and ${ }^{40} \mathrm{~K}$ (fermions) atoms, with a relevant ratio between the fermion and boson densities, $n_{F} / n_{B} \sim 0.5$, has been studied. For the homogeneous boson gas, a value $T_{c}=110 \mathrm{n} \mathrm{K}$ for the critical temperature of condensation has been chosen, in order to reproduce values of temperature and condensate fraction close to those reported in Ref. [5]. The corresponding boson density is $n_{B}=1.45 \times 10^{13} \mathrm{~cm}^{-3}$. This is a reasonable value for the average boson density obtained in the experiment of 


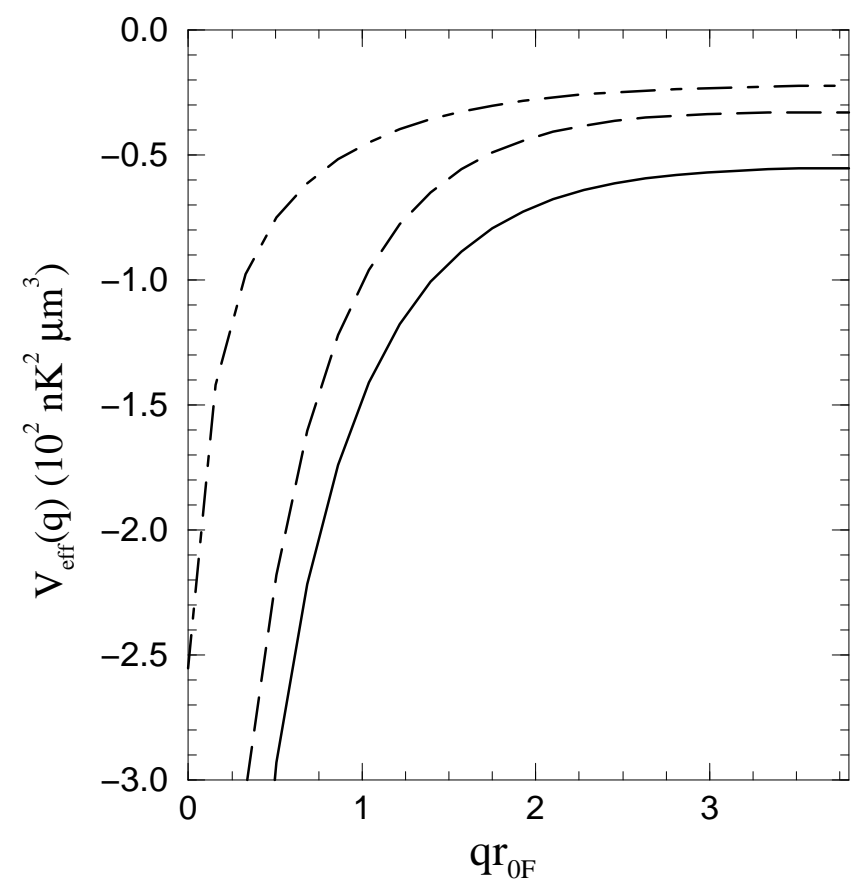

FIG. 1: Induced effective interaction between fermions as a function of the momentum transfer $q$, for a mixture of ${ }^{40} \mathrm{~K}$ and ${ }^{87} \mathrm{Rb}$ (solid line), $r_{0 F}$ is the mean spacing between fermions. The contributions from states with one virtual phonon ( dashed line) and with two virtual phonons ( dot-dashed line ) are also shown. The condensate fraction is $n_{B}^{0} / n_{B}=0.6$.

Ref. [5]. Moreover, for the boson-boson and boson-fermion scattering lengths, the values $a_{B B}=113 a_{0}$ and $a_{B F}=-330 a_{0}$ are used, where $a_{0}$ is the Bohr radius. The range of the condensate fractions considered in the present calculations is $0.4 \leq n_{B}^{0} / n_{B} \leq 0.8$, and the corresponding interval of temperatures is $46 \mathrm{n} \mathrm{K} \leq T \leq 87 \mathrm{n} \mathrm{K}$. With these values of the relevant parameters, the ratio between the Fermi velocity, defined as $v_{F}=\sqrt{2 \mu_{F}^{*} / m_{F}}$, and the sound velocity in the Bose gas, $c_{s}=\sqrt{\gamma n_{B}^{0} / m_{B}}$, turns out to be about 20. This value of $v_{F} / c_{s}$ is sufficiently large to justify the approximation of Eq. (20) to the effective interaction. Moreover, the Fermi temperature corresponding to the density $n_{F} \sim 0.5 n_{B}, T_{F} \sim 320 \mathrm{n} \mathrm{K}$, is high enough with respect to the temperatures in the interval considered, so that the Pauli principle is still operating.

The results for the effective interaction are discussed first. A simple inspection of Eq. (20) shows that the contributions from states with one virtual phonon and from states with two virtual phonons, approach the values $-\lambda^{2} n_{B}^{0}$ and $-\lambda^{2} n_{B}^{\prime}$, respectively, when $q \rightarrow \infty$.

In Fig. 1 the effective interaction of Eq. (20) is shown togheter with the separate contributions from one-phonon and two-phonon states, for $n_{B}^{0} / n_{B}=0.6$. We can see that the two contributions are of the same order of magnitude for $q \geq 0.5 r_{0 F}^{-1}$, with $r_{0 F}$ denoting the mean spacing of the Fermi atoms, and that the asymptotic values are practically reached already at $q \sim 1.5 r_{0 F}^{-1}$. This suggests that the contribution from states containing two virtual phonons cannot be neglected, unless the condensate depletion is very small.

In Fig. 2 the effective interaction is displayed for three different values of the condensate fraction. From Fig. 2 we can see that, in the considered range of $n_{B}^{0} / n_{B}$, for a fixed value of the boson density the magnitude of the effective interaction increases with the condensate 


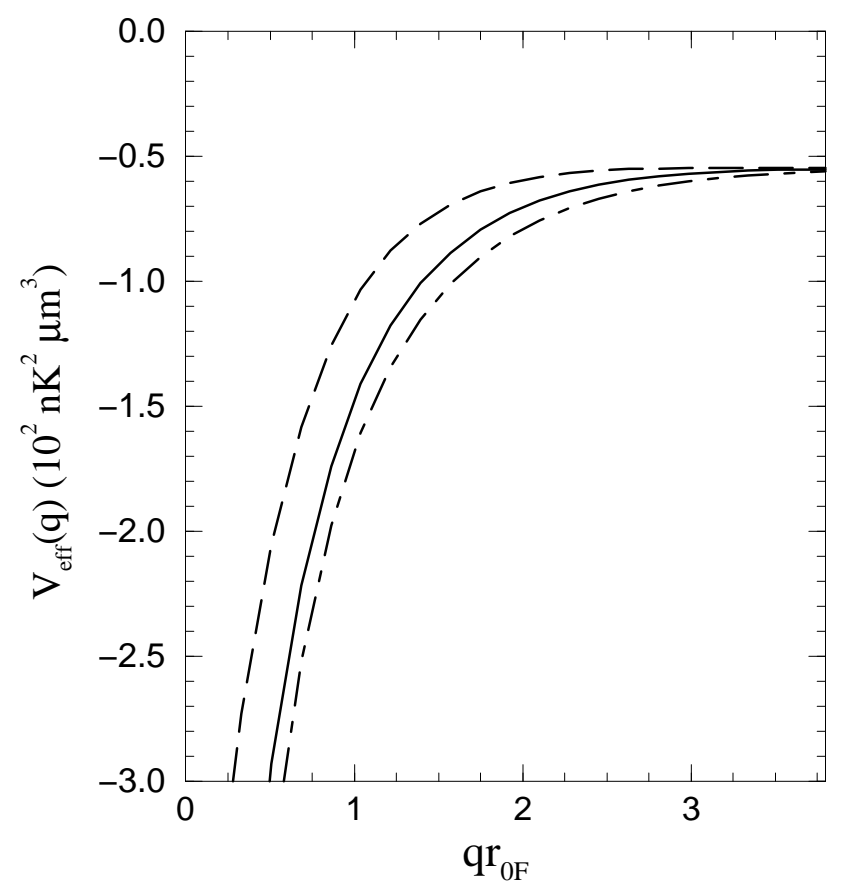

FIG. 2: Induced effective interaction between fermions as a function of the momentum transfer $q$, for three different values of the condensate fraction. The Bose-Fermi mixture is the same as in Fig. [1. Dashed line: $n_{B}^{0} / n_{B}=0.8$; solid line: $n_{B}^{0} / n_{B}=0.6$; dot-dashed line: $n_{B}^{0} / n_{B}=0.4$.

depletion, or, equivalently, with the temperature. This is due to the fact that the increase with the noncondensate fraction of the two-phonon contribution, overcomes the decrease with $n_{B}^{0}$ of the one-phonon contribution. However, for higher values of the condensate depletion, the increase of $V_{\text {eff }}(q)$ results considerably quenched.

Concerning the solutions of the gap equation (22), it should first be remarked that the fast approach of the effective interaction to an almost constant value makes the odd components $V_{l}\left(k, k_{1}\right)$ vanish rapidly at high momenta. As a consequence, for evaluating the integral of Eq. (22) it is not necessary to introduce any cut-off on high values of $k_{1}$. The explicit solutions of Eq. (22) display two general features for the physical situations considered: they give, for all values of $l$, a small energy gap, with respect to the effective chemical potential of the of the fermions, $\Delta_{l}(k) / \mu_{F}^{*} \sim 10^{-2}$, and they give a non vanishing energy gap only for a single value of $l$ at a time, without any coupling to the remaining components. The latter feature can be better understood by observing that, for small values of $\Delta_{l}(k)$, the most relevant contribution to the integral of Eq. (22) comes from a small domain about the effective Fermi momentum, $k_{F}^{*}=\sqrt{2 \mu_{F}^{*} m_{F}}$. This allows us to approximate all the quantities in the integrand, apart from $\xi_{k_{1}}$, by the values that they take at $k_{1}=k_{F}^{*}$. Thus, Eq. (22) turns into the following closed equation for each value of $k$

$$
\Delta_{l}(k)=-\frac{1}{4 \pi^{2}} m_{F} k_{F}^{*} V_{l}\left(k, k_{F}^{*}\right) \Delta_{l}\left(k_{F}^{*}\right) \frac{1}{\sqrt{\bar{\Delta}^{2}\left(k_{F}^{*}\right)}}\left[\frac{\pi}{2}+\arctan \left(\frac{\mu_{F}^{*}}{\sqrt{\bar{\Delta}^{2}\left(k_{F}^{*}\right)}}\right)\right],
$$

where the angle average $\bar{\Delta}^{2}\left(k_{F}^{*}\right)$, ( see Eq. (21) ), is given by

$$
\bar{\Delta}^{2}\left(k_{F}^{*}\right)=\sum_{l^{\prime}} \Delta_{l^{\prime}}^{2}\left(k_{F}^{*}\right) .
$$




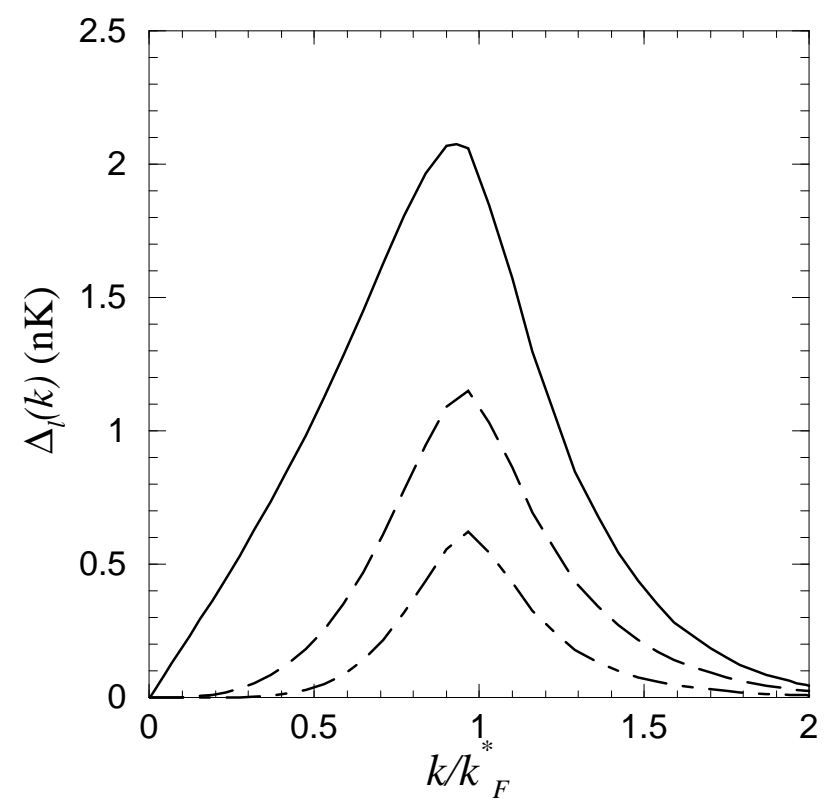

FIG. 3: Energy gap for different $l$-channels, as a function of the wave vector $k$ measured in units of $k_{F}^{*}=\sqrt{2 \mu_{F}^{*} m_{F}}$. Solid line: $l=1$; dashed line: $l=3$; dot-dashed line: $l=5$. The condensate fraction is $n_{B}^{0} / n_{B}=0.6$, corresponding to a temperature of $70 \mathrm{n} \mathrm{K}$. The Bose-Fermi mixture is the same as in Fig. 1]

It is easy to see that Eq. (23) can have nontrivial solutions only when $\Delta_{l^{\prime}}=0$ for $l^{\prime} \neq l$. In addition, it is worth noting that the energy gaps given by Eq. (23) represent a very satisfactory approximation to the numerical solutions of Eq. (22).

The solutions of Eq. (22) indicate that transitions to different superconducting phases could occur. Each phase is characterized by a nonisotropic pairing field, whose angular dependence is given by a specific spherical harmonic. The corresponding energy gap contains only one term $\Delta_{l}(k)$.

In Fig. 3 the energy gap is displayed for three different values of $l$ at a fixed condensate fraction ( or temperature ). The fast decrease of the energy gap with increasing $l$ corresponds to the analogous behavior of the effective interaction. While the peak shown by $\Delta_{l}(k)$ about $k=k_{F}^{*}$ is due to the sharp increase of the effective interaction when the transferred momentum appoaches zero. This can be directly seen from the approximate equation (23).

The behavior with the condensate fraction, of the energy gap for $l=1$ is reported in Fig. 4. All $l$-channels show a similar behavior. We see that, when $n_{B}^{0}$ decreases, or, equivalently, the temperature increases, the gap $\Delta_{1}(k)$ increases for all values of $k$. This feature essentially reflects the analogous behavior of the effective-interaction strength. However, the fermion gas becomes less and less degenerate when the temperature increases, so that, the corresponding decrease of the effective Fermi momentum tends to counterbalance the effect of the effective interaction. Actually, explicit calculations show that, for $T \sim 100 \mathrm{nK}$ corresponding to $n_{B}^{0} / n_{B} \sim 0.2$, the gap $\Delta_{1}(k)$ reverses its initial behavior. It should be observed that the approximation scheme proposed in this paper does not keep its validity for higher temperatures. In any case, the results of the present approach show that a superconducting phase in the $p$-wave channel can be achieved at temperatures $>100 \mathrm{nK}$. In Ref. [8] a critical temperature of $\sim 10^{-2} \mathrm{nK}$ was indicated for $p$-wave pairing in a mixture of ${ }^{6} \mathrm{Li}$ and ${ }^{87} \mathrm{Rb}$. 


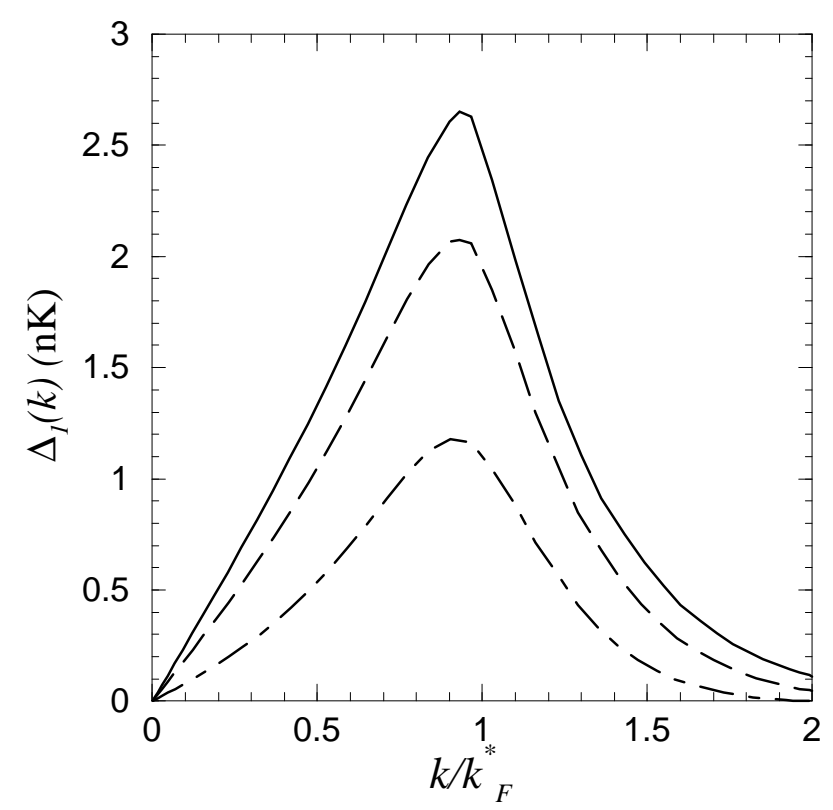

FIG. 4: Energy gap in the $l=1$ channel for different values of the condensate fraction. Solid line: $n_{B}^{0} / n_{B}=0.4(T=87 \mathrm{nK})$; dashed line: $n_{B}^{0} / n_{B}=0.6(T=70 \mathrm{n} \mathrm{K})$; dot-dashed line: $n_{B}^{0} / n_{B}=0.8(T=46 \mathrm{n} \mathrm{K})$. The Bose-Fermi mixture is the same as in Fig. 1]

For this mixture calculations performed within the present scheme and with the same values of parameters as in Ref. [8], show that the behavior of the energy gap qualitatively does not differ from that of Fig. 固. The deep discrepancy between the results of the present work and those of Ref. [8] should be ascribed to the fact that in the two approaches two opposite approximations have been used for the time dependence of the effective interaction. These approximations have already been discussed in Sec. IIB.

\section{SUMMARY AND CONCLUSIONS}

In a mixture of ultracold Fermi and Bose gases, an interaction between fermions is induced by the exchange of elementary excitations of the Bose gas. Due to this supplementary interaction, a superconducting phase can appear in the Fermi component of the mixture. The mechanism is analogous to that accounting for superconductivity in ordinary metals. In this paper, a procedure to determine the induced interaction and to assess the possibility of a phase transition has been proposed. For simplicity, a spatially homogeneous system has been considered. Both the gases have essentially been treated within a mean field approximation appropriate for the physical conditions realized in recent experiments: the values of temperature and densities are such that the Bogoliubov approximation for the Bose gas can be justified and the Fermi gas can be considered degenerate. Moreover, since the experiments concern gases confined by means of magnetic traps, in the present approach the atoms of both species have been considered completely polarized. This circumstance has allowed the bare fermion-fermion interaction to be neglected.

The same formalism, based on functional methods, has been used in all stages of the calculation. This allows us to introduce the various approximations to the effective interaction 
between fermions in a self-consistent way. In particular, the inclusion of the contributions from states containing two virtual phonons and the static approximation to its time dependence. This is more appropriate for the physical situations of actual experiments than the usual instantaneous approximation. Both terms of the interaction, with one and two virtual phonons, are attractive and of the same order of magnitude. They can give rise to the pairing of correlated couples of fermions with different values of the center of mass momentum. In this paper, only the pairing field with vanishing center of mass momentum has been considered, thus preserving the invariance of the system under space translations. Since the paired fermions lie in the same magnetic state, the pairing field is constrained to be an odd function of their relative momentum. Then, it contains only odd $-l$ components, when expanded in spherical harmonics. A particular feature of the corresponding gap equations is that they show solutions with only one non vanishing partial wave at a time. Then, different nonisotropic phases may occur in the Fermi component of the mixture, with the pairing fields decreasing their strength when their multipolarity increases. It is worth noting that the energy gaps increase with the temperature until a value of $T \sim 100 \mathrm{nK}$, corresponding to a high depletion of the Bose condensate $n_{B}^{0} / n_{B} \sim 0.2$. The approximations introduced in the present approach become less reliable for higher values of the temperature, for the values of boson and fermion densities assumed here. Nevertheless, we can expect that a superconducting phase can occur in a Bose-Fermi mixture at $T>100 \mathrm{nK}$. This value is a relevant fraction of the Fermi temperature.

\section{Acknowledgments}

I am grateful to M. Modugno, M. Baldo and H.-J. Schulze for valuable discussions. I would also like to thank A. Dellafiore for a careful reading of the manuscript.

[1] J. Bardeen, G. Baym, and D. Pines, Phys. Rev. 156, 207 (1967).

[2] A. G. Truscott, K. E. Strecker, W. I. McAlexander, G. B. Partridge, and R. G. Hulet, Science 291, 2570 (2001).

[3] F. Schreck, L. Khaykovich, K. L. Corwin, G. Ferrari, T. Bourdel, J. Cubizolles, and C. Salomon, Phys. Rev. Lett. 87, 080403 (2001).

[4] Z. Hadzibabic, C. A. Stan, K. Dieckmann, S. Gupta, M. W. Zwierlein, A. Görlitz, and W. Ketterle, Phys. Rev. Lett. 88, 160401 (2002).

[5] G. Roati, F. Riboli, G. Modugno, and M. Inguscio, Phys. Rev. Lett. 89, 150403 (2002); G. Modugno, G. Roati, F. Riboli, F. Ferlaino, R. J. Brecha, and M. Inguscio, Science 297, 2240 (2002).

[6] M. J. Bijlsma, B. A. Heringa, and H. T. C. Stoof, Phys. Rev. A 61053601 (2000).

[7] H. Heiselberg, C. J. Pethick, H. Smith, and L. Viverit, Phys. Rev. Lett. 852418 (2000).

[8] D. V. Efremov and L. Viverit, Phys. Rev. B 65, 134519 (2002).

[9] L. Viverit, Phys. Rev. A 66, 023605 (2002).

[10] J. W. Negele and H. Orland, Quantum Many-Particle Systems (Addison-Wesley, New York, 1988).

[11] H. T. C. Stoof, in Coherent atomic matter waves, Proceedings of the Les Houches Summer School, Session 72, edited by R. Kaiser, C. Westbrook, and F. David (Springer-Verlag, Hei- 
delberg, 2001), p. 219.

[12] A. L. Fetter and J. D. Walecka, Quantum Theory of Many-Particle Systems (McGraw-Hill, New York, 1971).

[13] R. L. Stratonovich, Sov. Phys. Dokl. 2, 416 (1958).

[14] J. Hubbard, Phys. Rev. Lett. 3, 77 (1959).

[15] A. I. Larkin and Yu. N. Ovchinnikov, Sov. Phys. JEPT 20, 762 (1965); P. Fulde and R. A. Ferrel, Phys. Rev. 135, A550 (1964). 